\title{
Innovación en salud: atención primaria con participación de la comunidad en Punta Indio, Argentina
}

\author{
Fernández, Marcela Indiana*
}

\section{Resumen}

En este artículo se expone la experiencia del Programa de Salud del Municipio de Punta Indio en Argentina, que está en ejecución desde hace cuatro años, porque permite dar cuenta de elementos que se consideran innovadores con respecto a la atención primaria por el componente que reviste la participación de la comunidad, como elemento movilizador de las normas prácticas de gestión y la incorporación de servicios que descansan en un recurso humano adecuadamente capacitador y que ha sido preparado para el trabajo en equipo. Con ello se demuestra que cuando las propuestas en salud surgen del trabajo conjunto de la comunidad con equipos técnicos especializados y los líderes políticos interesados en el tema de la atención de la salud, los resultados que se alcanzan son óptimos.

Palabras clave: Atención primaria, participación de la comunidad, Punta Indio, Argentina, servicios de salud, política municipal.

\section{Recibido: 00-07-27. Aceptado: 00-09-20}

Investigadora del Programa ALDEA XXI. Centro de Estudios e Investigaciones. Universidad Nacional de Quilmes. Profesora Adjunta. Diplomatura en Ciencias Sociales. U.N.Q. Jefe de Trabajos Prácticos. Departamentos de Historia y Geografia. Facultad de Filosolia y Letras. Universidad de Buenos Aires.

Una parte substancial de este articulo corresponde a la ponencia presentada en las Primeras Jornadas de Politicas Sociales del MERCOSUR: Hacia una estrategia regional para el ordenamiento, construcción y evaluación de las politicas sociales. Buenos Aires, 28 al 30 de junio de 2000. 


\title{
Innovations in Salud Primary Attention and Cummunity Participation in Punta Indio, Argentina
}

\begin{abstract}
This article explains certain experiences in the Punta Indio Municipal Health Program in Argentina, which has been in operation for four years, because it permits us to understand the elements which are considered innovative with respect to primary attention through participation on the part of the community, which is considered to be the mobilizing element in the establishment of operational norms and the incorporation of services which depend on well trained human resources that have been prepared as a team. In this way we demonstrate the health proposal which arose through the integrated work of the community and the specialized teams, as well as the political leaders interested in this theme of public health attention: The results obtained are optimum.
\end{abstract}

Key words: Attention primary, community participation, Punta Indio, Argentina.

\section{Introducción}

"Indio Sano", el Programa de Salud del Municipio de Punta Indio en Argentina, que está ejecutándose desde hace cuatro años, cuenta con interesantes elementos que podríamos considerar "innovadores". Dicho programa se genera a partir de las demandas de la población local, en un proceso inédito de participación ciudadana amplia en el diseño del "modelo de salud" que se quería para el municipio. Así, políticos, técnicos y vecinos articularon un proyecto innovador de origen local, "médico y enfermera de familia", con la oferta clásica de recursos de origen provincial, a partir de una "revolución" en la que se definió priorizar como centro de las políticas del área a la "prevención y educación para la salud", garantizando simultáneamente la atención de la enfermedad. Se monta sobre un proyecto político fundacional para la comunidad de Punta Indio que en 1994, logra la separación política del municipio de Magdalena al cual pertenecía.

Las propuestas del gobierno municipal, en coincidencia con principios que se apuntan en la Ley de Creación del Municipio (11.584/94), están profundamente vinculadas con la participación, materializada en nuevas prácticas e instituciones para la comunidad organizada y con nuevas modalidades para la gestión en el ámbito municipal.

El programa directriz del área de Salud, conformado por un subprograma vertebral "Médico y Enfermera de Familia" y otros subprogramas que abordan cuestiones especificas, es una respuesta a una demanda histórica que la población logra legitimar por medio de la participa- 
ción masiva y directa: la presencia de profesionales médicos en forma permanente en las localidades de la jurisdicción (el médico de cabecera/familia). Su diseño responde a un cambio en la concepción del binomio salud-enfermedad. En el modelo de atención dominante, el énfasis está puesto en la atención de la enfermedad (instituciones, aparatología, medicación, costos elevados, sujeto pasivo) mientras que la propuesta se basa en la atención primaria y en la preservación y educación para la salud. Este cambio que implica educar y crear conciencia, restituir el protagonismo del sujeto en la práctica médica, cambiar el vínculo médico-paciente, etc. exige de nuevas prácticas de gestión para materializarlo. Por ej. el diseño del servicio del "médico de cabecera" se basa en un recurso humano muy capacitado y caro, pero utilizado intensivamente, predomina el trabajo en equipo sin estructuras de coordinación, pero en contacto permanente.

\section{Gestión local e innovación}

¿Existen transformaciones significativas en los modelos de gestión municipal? ¿qué características poseen? Si bien no hay acuerdo cerrado en relación con las especificidades de un nuevo modelo, pueden verificarse "esfuerzos innovativos" en la gestión local, que van desde la aplicación de nuevas técnicas (sistemas de información geográfica, modificación de sistemas de administración de rentas o de racionalización de personal, etc.) de impacto sectorial, a la implementación de acciones de carácter más global, como por ej. el planeamiento estratégico, el presupuesto participativo, la incorporación de ONGs y de la ciudadanía en la gestión de problemas sociales; los programas integrales o sectoriales de capacitación, etc. También son interesantes las acciones asociativas de base territorial que surgen en determinados ámbitos para beneficio de un conjunto de gobiernos locales (consorcios, corredores productivos, etc.).

$\mathrm{Si}$ se examinan las tareas/acciones/iniciativas que se desarrollan en los ámbitos de los gobiernos locales, hoy los municipios se ocupan, por un lado, de sus viejas y tradicionales funciones pero con una perspectiva distinta. Se encargan por ej. de sostener las demandas sociales involucrando a los beneficiarios en las acciones de los programas, buscando ampliar los compromisos con la población y soslayar el asistencialismo tradicional; se encargan de brindar servicios para un vecino que ahora se valora como ciudadano-cliente-consumidor al que se le ofrece calidad en las prestaciones, transparencia en la administración, etc. Por otro lado, encaran funciones nuevas con el objetivo básico de promover el desarrollo local favoreciendo el despliegue de las "potencialidades" económicas, sociales y políticas de la comunidad localizada.

¿Cómo desarrollan los municipios estas iniciativas? Actuando con "estrategias y herramientas innovadoras" que les permiten movilizar a una gran cantidad de actores del ámbito local (sector privado, ONGs, organismos provinciales, nacionales, internacionales, sociedad civil, etc.) a los que es necesario involucrar para gestar el proceso. De acuerdo con esta lógica, la participación de los actores a través de diversos mecanismos, debería generarse y propiciarse para utilizar los recursos con mayor racionalidad, mejorar la calidad de 
los servicios, aumentar la transparencia, cambiar la actitud psicosocial de los sujetos, favorecer la democratización disminuyendo el peso del clientelismo político, crear nuevas culturas, etc.

Una nueva imagen del municipio argentino y latinoamericano se va con. formando, a partir de los ochenta; es la de "... un territorio donde se realizan un conjunto de intercambios y actividades de carácter económico y social, que pueden ser susceptibles de incentivación y de promoción por parte de la municipalidad". (Victory, 1997:17). Este carácter del gobierno local ha producido una ruptura de los esquemas típicos de administración y demanda otras herramientas, por ej. nuevos actores son convocados a participar en un gobierno que aparece como el referente inmediato de las demandas concretas de la gente. Como institución que busca dar respuestas a los problemas que se le plantean tiene que asumir plenamente nuevas competencias y funciones que le transfieren el orden nacional y el provincial, a través de sucesivos procesos de "descentralización"1.

Desde distintos ámbitos se señala que los "proyectos/acciones/iniciativas innovadoras" permitirian al gobierno munici- pal, en un contexto de crecimiento/presión de las demandas al sector público y de restricciones presupuestarias también crecientes, derivadas ambas de problemas económicos y sociales críticos que afectan al conjunto de la población, "hacer las cosas de siempre pero de otra manera", vale decir cumplir con sus funciones tradicionales apelando a nuevas herramientas de gestión y "hacer cosas nuevas", en la dirección de las nuevas funciones.

¿Qué aparece como innovador en los gobiernos locales? Por un lado, aparecen las iniciativas que implican encarar las nuevas funciones (desarrollo económico local, promoción de empleo, preservación del ambiente, promoción social). Por otro lado, aparecen experiencias puntuales que incorporan a la administración gubernamental lógicas empresariales (tercerización de funciones; adopción de criterios de eficiencia y de calidad en la prestación de servicios, de transparencia en la administración, etc.), con el objetivo básico de administrar mejor los recursos en una economía de escasez. Hacia el interior de la administración municipal, se reconocen como innovadoras a las iniciativas que plantean la articulación de acciones, pudiendo involucrar áreas de ad-

Los gobiernos locales reciben "ofentas de recursos", provenientes de distinto tipo de instituciones o de otros niveles a los que acceden por medio de "proyectos" que implican preparación planificación de propuestas que cumplan con los requerimientos de acceso, los plazos de ejecución y el control de la gestión. Esta modalidad de acceso revela: a) problemas estructurales vinculados a los cuadros profesionales imprescindibles para la formulación técnica de los proyectos; b) la ausencia de participación social/comunitaria. Se entiende que la participación limitada de la población deriva de prácticas históricas de las administraciones que han contribuido a crear la "cultura de recibir lo que se da" como mera asistencia para resolver las emergencias, que a menudo se estructura a través del clientelismo político. 
los servicios, aumentar la transparencia, cambiar la actitud psicosocial de los sujetos, favorecer la democratización disminuyendo el peso del clientelismo político, crear nuevas culturas, etc.

Una nueva imagen del municipio argentino y latinoamericano se va conformando, a partir de los ochenta; es la de "... un territorio donde se realizan un conjunto de intercambios y actividades de carácter económico y social, que pueden ser susceptibles de incentivación y de promoción por parte de la municipalidad". (Victory, 1997:17). Este carácter del gobierno local ha producido una ruptura de los esquemas típicos de administración y demanda otras herramientas, por ej. nuevos actores son convocados a participar en un gobierno que aparece como el referente inmediato de las demandas concretas de la gente. Como institución que busca dar respuestas a los problemas que se le plantean tiene que asumir plenamente nuevas competencias y funciones que le transfieren el orden nacional y el provincial, a través de sucesivos procesos de "descentralización"1.

Desde distintos ámbitos se señala que los "proyectos/acciones/iniciativas innovadoras" permitirian al gobierno munici- pal, en un contexto de crecimiento/presión de las demandas al sector público y de restricciones presupuestarias también crecientes, derivadas ambas de problemas económicos y sociales criticos que afectan al conjunto de la población, "hacer las cosas de siempre pero de otra manera", vale decir cumplir con sus funciones tradicionales apelando a nuevas herramientas de gestion y "hacer cosas nuevas", en la dirección de las nuevas funciones.

¿Qué aparece como innovador en los gobiernos locales? Por un lado, aparecen las iniciativas que implican encarar las nuevas funciones (desarrollo económico local, promoción de empleo, preservación del ambiente, promoción social). Por otro lado, aparecen experiencias puntuales que incorporan a la administración gubernamental lógicas empresariales (tercerización de funciones; adopción de criterios de eficiencia y de calidad en la prestación de servicios, de transparencia en la administración, etc.), con el objetivo básico de administrar mejor los recursos en una economía de escasez. Hacia el interior de la administración municipal, se reconocen como innovadoras a las iniciativas que plantean la articulación de acciones, pudiendo involucrar áreas de ad-

1 Los gobiernos locales reciben "ofertas de recursos", provenientes de distinto tipo de instituciones 0 de otros niveles a los que acceden por medio de "proyectos" que implican preparación/ planificación de propuestas que cumplan con los requerimientos de acceso, los plazos de ejecución y el control de la gestión. Esta modalidad de acceso revela: a) problemas estructurales vinculados a los cuadros profesionales imprescindibles para la formulación técnica de los proyectos; b) la ausencia de participación social/comunitaria. Se entiende que la participación limitada de la población deriva de prácticas históricas de las administraciones que han contribuido a crear la "cultura de recibir lo que se da" como mera asistencia para resolver las emergencias, que a menudo se estructura a través del clientelismo político. 
ministración, niveles de gobierno, variedad de actores, etc. Por último, la innovación se asocia con el desarrollo de mecanismos de participación ciudadana (sociedad civil, tercer sector, beneficiarios de programas, etc.) muy valorados (al menos en el discurso) por los dirigentes políticos y reivindicados por la población, no siempre con resultados exitosos.

A partir de la consulta de experiencias innovadoras en el Banco de Experiencias Locales (UNQ), trabajos de campo, estudios de caso y sugerencias de informantes calificados, se observa que las nuevas formas de gestión se van desarrollando sin un patrón preciso dentro de las administraciones locales, en áreas generadas para las nuevas funciones (desarrollo local, promoción de empleo, etc.) y aún en sectores tradicionales típicos de la administración (Obras Públicas, Acción Social, etc.) $)^{2}$.

En este sentido, ¿cuáles son los efectos que generan estas nuevas prácticas en el ámbito local?, ¿qué acciones pueden desarrollarse para mejorar situaciones que se consideran deficitarias?, ¿qué recursos materiales y humanos pueden movilizarse?, ¿cómo se promueve, garantiza y materializa la participación amplia y democrática de los ciudadanos en la gestión local?, ¿se transfieren las iniciativas innovadoras a otros ámbitos de la estructura administrativa del gobierno o quedan encapsuladas en los ámbitos en los que se originan?
Examinaremos algunas de estas cuestiones a partir de la consideración del "Indio Sano", el Programa de Salud del Municipio de Punta Indio (Buenos Aires) que está ejecutándose desde hace cuatro años, a partir de la creación de esta nueva administración local en la provincia de Buenos Aires.

\section{Un "nuevo municipio" para Punta Indio. El lugar de la salud en la preocupación comunitaria}

El programa de salud del municipio se genera a partir de las demandas de la población local, en un proceso inédito de participación ciudadana amplia en el diseño del "modelo de salud" que se quería para el municipio. Políticos, técnicos y vecinos articularon un proyecto innovador de origen local, "médico y enfermera de familia", con la oferta clásica de recursos de origen provincial, a partir de una "revolución": que tiene como objetivo priorizar como centro de las políticas del área a la "prevención y educación para la salud", garantizando la atención de la enfermedad.

En este contexto, la participación entendida como el involucramiento de los vecinos/ciudadanos en el diseño del programa de salud, con atribuciones plenas y vínculos estrechos con los equipos técnicos, tenía varios propósitos para la gestión. Por un lado, asegurar mecanismos ción teórica. Estudio de casos" Programa ALDEA XXI. Centro de Estudios e Investigaciones. Universidad Nacional de Quilmes. 
de transparencia en la asignación de los recursos, propiciar un uso intensivo de los mismos; atender particularmente a la calidad en los servicios ofrecidos capacitando al personal de la institución, tercerizando actividades., etc. Por otro lado, materializando la participación ciudadana "amplia y democrática" en el diseño institucional del municipio y del programa de salud en particular, el gobierno local pretendía, responder a los vecinos legitimando una vieja demanda de la comunidad del nuevo municipio, históricamente "desatendida" en este y otros temas por el Municipio de Magdalena a cuya jurisdicción pertenecia, reforzando la pertenencia a la nueva jurisdicción en la etapa fundacional de la "ciudadanía local" y, a la $v e z$, disminuir las diferencias entre áreas urbanas y rurales en lo relativo a la asignación de recursos y calidad de servicios. Esta había sido una demanda siempre postergada de las comunidades rurales de Punta Indio (varias pequeñas localidades y población rural dispersa), desfavorecidas por una alta concentración de población en la ciudad de Verónica, hoy cabecera del Municipio, que captaba la mayor parte de los recursos disponibles.

Indio Sano, el Programa "estrella del municipio", se monta sobre un proyecto politico fundacional para la comunidad de Punta Indio que en 1994, logra la separación política del municipio de Magdalena al cual pertenecía. Las propuestas del gobierno local, en coincidencia con principios que se apuntan en la Ley de Creación del Municipio (11.584/94), están profundamente vinculadas con la participación, materializada en nuevas prácticas e instituciones para la comunidad organizada.
El programa universal de salud "Indio Sano" (Licciardi, 2000) reunía por los menos dos aspectos atractivos como para transformarse en un caso a analizar en relación con las "prácticas innovadoras" en la gestión local.

1) Por un lado, la participación de la comunidad en el diseño y posterior sostenimiento de algunas acciones del programa de salud tomó una dimensión particularmente notoria. La participación directa (una verdadera movilización popular) de la comunidad a través de asambleas públicas y de comisiones permanentes de trabajo, estaba en consonancia con las ideas que los líderes políticos locales tenían sobre el manejo de la política en el ámbito municipal "gobemar con la gente". Esta participación amplia valorizó enormemente a los sujetos en tanto personas y ciudadanos, que asumían compromisos en relación con sus propios intereses y los de su comunidad. Todo este proceso generó una "nueva ciudadania para el nuevo municipio" (Bertini, 1995; Groisman, 1988; Martínez, 1995; Kliksber, 1992).

2) El propio diseño utilizó una importante variedad de nuevas herramientas de gestión provenientes de las estrategias y prácticas implementadas por el sector privado (Garcia Delgado, 1997; Kliksberg, 1992; Bustelo, 1995; Fernández, 1997; Passalacqua, 1994; Pérez, 1991). Entre ellas destacamos:

a) Satisfacción al cliente: los beneficiarios del programa que estructura el sistema público universal de salud son tomados en calidad de clientes/consumidores/usuarios a los que debe satisfacerse, brindándoles la mejor asistencia. 
b) Calidad del servicio: la prestación del servicio se encara con normas de calidad que mejoran los resultados de la propia actividad privada (por ej. se pretende alcanzar estandares internacionales sobre características de los servicios recomendados por UNICEF-OMS/"Hospital Amigo de los Niños"); (Ministerio de Salud de la Provincia de Buenos Aires, 1996).

c) Tercerización de servicios: convocatoria al sector privado para la oferta de servicios; el Hospital Municipal de reciente creación tiene gestión privada integral, con control municipal; el Director y personal médico/entermeros, son empleados de planta permanente de la administración;

d) Política laboral particular: el sistema se organiza a partir de un uso muy intensivo de recursos humanos muy calificados y caros; la política laboral del sector salud implicó la reconversión de todo el personal del sistema y la capacitación permanente en los puestos de trabajo.

e) Atención a la demanda: el programa de salud es una respuesta técnica a la demanda comunitaria, a partir de prioridades surgidas de la propia comunidad;

f) Transparencia en la gestión: por un lado, se garantiza un acceso franco a la información sobre las inversiones realizadas en el sector y se impulsa la producción de información para la toma de decisiones; por otro lado, se establecen diversos mecanismos para el control de las acciones;

3) Por último, el programa de salud incorporó otras iniciativas consideradas innovadoras: a) Atención de la población con objetivos federales: la oferta del servicio se organiza con la intención de una "reparación histórica" que lleva a disminuir las diferencias al interior de la jurisdicción del municipio (en relación con la universalidad del servicio, el acceso tiene que estar garantizado para todos, en condiciones similares, sin que existan "privilegios por la localización, por ej. en la ciudad de Verónica).

b) Articulación de acciones entre áreas de la administración municipal: el programa de salud maneja una parte importante de recursos propios, pero además, sus programas se articulan con los de desarrollo social y educación: se comparte infraestructura, diseño de acciones, trabajo del personal técnico-profesional, etc. Por ej. el Area de Sistemas del municipio apoya con asesoramiento técnico y con información, el trabajo de otros sectores, entre ellos salud.

Una parte importante de los programas y acciones que se desarrollan en el gobierno local, en Punta Indio, revela transformaciones importantes en el modelo de gestión; este cambio ha ocurrido porque, a pesar de algunas resistencias, existe enorme consenso y legitimidad politica para la gestión innovadora que impulsan las actuales autoridades del municipio.

\section{La atención de la salud desde una perspectiva innovadora}

"Indio Sano" (Licciardi, 2000), el programa directriz del área de Salud del Municipio, está conformado por un sub- 
programa central/básico/vertebral "Médico y Enfermera de Familia" y otros once subprogramas dirigidos a atender cuestiones especificas.

Tiene una gestación muy particular; surge de los intereses prioritarios de la población autoconvocada para participar en las jornadas programáticas para la construcción del nuevo municipio. En este sentido, es una respuesta a una demanda histórica que la población logra legitimar a través de la participación masiva y directa: la presencia de profesionales médicos en forma permanente en las localidades de la jurisdicción (el médico de cabecera/familia).

El diseño responde a un cambio en la concepción del binomio salud-enfermedad. En el modelo de atención dominante, el énfasis está puesto en la atención de la enfermedad (instituciones, aparatología, medicación, costos elevados, sujeto pasivo) mientras que la propuesta de Punta Indio se basa en la atención primaria y en la preservación y educación para la salud. Este cambio implica educar y crear conciencia, restituir el protagonismo del sujeto en la práctica médi$\mathrm{ca}$, cambiar el vínculo médico-paciente, etc.; exige de las nuevas prácticas de gestión para materializarlo (por ej. el diseño del servicio del "médico de cabecera" se basa en un recurso humano muy capacitado y caro, pero utilizado intensivamente, predomina el trabajo en equipo sin estructuras de coordinación, pero en contacto permanente; etc.).

Estas iniciativas que prosperan particularmente en el ámbito de la gestión de la salud no constituyen un fenómeno aislado que tiene lugar en la Dirección de
Salud y Acción Social del Municipio, deben enmarcarse en una gestión innovadora que atraviesa toda la administración local. Todo el gobierno aparece como innovador al definir desde cero, un diseño institucional que incorpora una nueva concepción de la gestión, priorizando y delimitando el conjunto de funciones que estarían a cargo del municipio, asumiéndolas como "indelegables". Por ej. al definirse la prestación de la salud como una función indelegable del municipio el gobierno local se hace cargo directamente de la organización del sistema.

El punto de partida que posibilita el cambio del diseño institucional y del modelo de gestión resulta de una oportunidad extraordinaria, que pudo aprovecharse con resultados beneficiosos para la comunidad; este hecho extraordinario fue la creación del municipio de Punta Indio en la Provincia de Buenos Aires, por Ley 11.584/94, a partir de la subdivisión de Magdalena.

La "gesta de la autonomia", como la llaman los vecinos, generó una gran movilización popular, por fuera de instituciones más o menos representativas (partidos políticos, sociedades de fomento, otras ONGs, etc.) y una intención de participación en la definición de los problemas de la comunidad que se aprovechó políticamente para crear consenso en lo relativo a la organización del nuevo municipio. La participación de la comunidad en la gestión local constituía una deuda histórica para Verónica y otras localidades que hoy forman parte del municipio, alejadas/ajenas de la administración municipal ubicada en la ciudad de Magdalena. Abriendo espacios a la parti- 
cipación se respondía a una demanda histórica y se propiciaba el involucramiento.

\section{1. Punta Indio: El espacio de la participación en la gestión local $\left.\right|^{3}$}

Las iniciativas que propician un aumento de la participación de la población en cuestiones relacionadas con las "demandas sociales" implican la construcción de nuevos espacios institucionales aparecen como cambios innovadores porque empiezan a considerar al demandante y a la problemática en la que está inmerso (Mendicoa, 1997; Bertini, 1995).

¿Cómo se instrumenta la participación de la población en los programas o acciones que se impulsan desde el gobierno local? En Buenos Aires suele canalizarse a través de las entidades intermedias o de bien público, privilegiando a las "sociedades de fomento barriales"4.
¿Qué ocurrió en Punta indio? El 6 de diciembre de 1994 la Legislatura bonaerense sanciona la Ley 11.584 , de Creación del Municipio de Punta Indio, después de cerca de 40 años de iniciativas y peticiones para alcanzar la separación del Municipio de Magdalena del que formaban parte. El nuevo municipio hereda una estructura administrativa que va a rediseñar para cumplir con las demandas de su comunidad, optando por un proceso de construcción de sus instituciones "desde abajo", cogestado con la población que encuentra formas de participación directa por fuera de canales instituidos, como por ej. las viejas sociedades de fomento barriales.

En palabras de su Intendente Dr. L. Colabianchi, la comunidad de Punta Indio, a partir de la convicción de que se podia "vivir con lo propio" 5 había alcanzado una notable madurez cívica y asi lo demostró, primero motivada por la lucha

Las opiniones del Intendente Dr. Luis Colabianchi corresponden a una larga entrevista realizada en febrero de 2000 en Verónica (Buenos Aires).

Las sociedades de fomento reconocidas como tales en los municipios tienen personería jurídica que las legitima y una jurisdicción territorial exclusiva (cubren el municipio); representan los intereses de los vecinos desde un lugar pretendidamente desvinculado de lo político, a la vez que como los interlocutores válidos que reconoce el gobierno local, canalizan las demandas de su jurisdicción. Desde este lugar el gobierno local establece las relaciones con la sociedad civil; se canalizan las demandas, se brinda asesoramiento general, se convoca para la ejecución de acciones y, en algunos casos, se descentraliza la gestión de una parte (muy menor) de los recursos disponibles.

5 Esta era la percepción de la comunidad, durante largo tiempo se había sentido muy relegada a raiz de los "olvidos sistemátiços" de la administración de Magdalena. Estas localidades ubicadas en la zona sur del viejo municipio soportaron la precariedad de caminos y deficientes medios de comuniçación. Las distancias importantes con la ciudad cabecera, trajeron un fuerte aislamiento que impuso el localismo como forma de vida, originando además, los reclamos autonomistas del sur. 
en pro de la "autonomía", luego ocupando un espacio importante a la hora de "delinear las estrategias fundacionales de un nuevo modelo municipal" que se esquematizó en el "Acuerdo-Compromiso entre los Partidos Políticos, Representantes del Pueblo y Entídades del Futuro Municipio", firmado el 8 de abril de 1994, frente a la necesidad de "convenir el régimen y funcionamiento de la comuna a crearse". En este documento se consensuaba un nuevo modelo/diseño de institución municipal que se apoyaba en dos aspectos prioritarios: una democracia participativa y un nuevo modelo de organización institucional.

a) La democracia participativa implicaba el protagonismo popular pleno y amplia injerencia de los vecinos en los asuntos municipales que se materializaría a través de diversos mecanismos e instituciones; el objetivo básico era incorporar en forma activa a las organizaciones intermedias y a la comunidad; quedaba el compromiso de creación de los "consejos vecinales" para el desarrollo de actividades comunitarias que se requiriesen.

El gobierno garantizaria la elección de los delegados municipales, en forma directa por la población residente en cada jurisdicción y los partidos políticos se comprometian a integrarlas listas de concejales con vecinos de todas las localida$\operatorname{des}^{6}$. Este concepto de democracia participativa se completaba con el compromiso de aceptar sugerencias de entidades intermedias y la comunidad para profundizar los cursos de acción que estaban surgiendo, en la oportunidad de discutir el funcionamiento, los roles y las atribuciones del nuevo municipio.

Varias de estas alternativas de participación fueron cristalizando a partir de la creación del nuevo gobierno local. Para febrero del 95, se convocó a la Primera Jornada Programática para el Nuevo Municipio, alli se reunieron instituciones y vecinos en igualdad de condiciones para materializar los intereses de la comunidad, hacer propuestas, identificar demandas, etc. En estas grandes asambleas públicas se fueron conformando grupos de trabajo por áreas de interés para el tratamiento de los temas prioritarios que fueron adquiriendo importancia y legitimidad, tal es el caso de los Consejos Vecinales Locales de Salud que van a trabajar especialmente en las propuestas para el área, a la par de los equipos técnicos.

Por otro lado, la mayor parte de los acuerdos previos se materializó institucionalmente a través de ordenanzas del Consejo Deliberante sustentadas muchas de ellas en la producción de las comisiones de trabajo, por ej. de los consejos vecinales de salud, que se fueron conformando a partir de las Jornadas Programáticas y de las reuniones sucesivas a las que se comprometian los vecinos nucleados en los consejos. menor tamaño y áreas rurales sustenta la acusación de centralismo que recibe el municipio bonaerense, tiene relación dirrecta con la distribución de la población, muy concentrada en la localidad cabecera. 
En mayo del 95 se eligieron las primeras autoridades de municipio y en agosto, por primera vez en la Provincia de Buenos Aires, dos pequeñas localidades, Pipinas y Punta Indio, tuvieron la posibilidad de elegir por voto directo al "Delegado Municipal" que deja de ser un "delegado" para transformarse en un "representante" de estas comunidades "históricamente relegadas" por la lógica de la administración imperante.

En la primera sesión del Consejo Deliberante (fines del 95), junto con el conjunto de reglamentaciones que organizan la institución municipal, se sanciona la ordenanza de creación de la Comunidad Organizada ( $\left.\mathrm{N}^{\circ} 25 / \mathrm{feb} .96\right)$ institucionalizando los Consejos de Gestión Vecinal, compuestos por representantes de entidades intermedias y/o miembros de la comunidad con funciones relacionadas con la captación de problemas y demandas de la población y con el asesoramiento sobre cuestiones de interés comunitario; estos consejos sirven para articular relaciones entre la sociedad civil y el sector público. A la par que los consejos, se crea la Banca Honoraria en el Consejo Deliberante, a la que puede acceder la comunidad cuando quiere hacer oír sus demandas o sugerencias en temas de interés, se trata de una presencia con voz pero sin voto.

Estas acciones corresponden a una primera etapa de trabajo en la administración local a la que llaman "fundacional"; en ella, la participación fue muy amplia porque la motivación para la construcción del municipio era muy fuerte; ya han pasado algunos años $y$, como era de esperar, la participación ha disminuido.
Sin embargo, el diseño de los programas, por ej. en el área de salud, convoca en forma permanente a la comunidad en general $o$ a algunas de sus instituciones en acciones concretas vinculadas con la educación, la promoción de la salud, la difusión de temas de interés, etc.

b) La institución municipal se concebia como una "empresa social prestadora de servicios públicos indelegables"; el resto de los servicios se prestaria a partir de formas asociativas legalmente constituidas, concesiones, privatizaciones $u$ otras modalidades permitidas por la Ley Orgánica de las Municipalidades y el Reglamento de Contabilidad de la Provincia; en condiciones de igualdad de calidad y precios, se establecian prioridades para las empresas residentes en el municipio.

La nueva administración tendría particular cuidado en el tema del gasto público, que debería formularse garantizando eficiencia y calidad, a la vez que la austeridad necesaria para cumplir con los objetivos comunitarios. Las prioridades del gobierno estarían vinculadas con las necesidades reales de la población de todas las localidades y áreas rurales, con la intención de reparar la postergación de muchas áreas, por lo tanto, el presupuesto debería materializar el federalismo político, económico y social.

En lo relativo a las fuentes de financiamiento de la futura administración (tasas municipales)se propiciaria una política de ingresos genuinos orientada a favorecer el desarrollo y el crecimiento regional y a disminuir la presión fiscal.

Entre las funciones indelegables el gobierno local garantizaria el "cumpli- 
miento real y efectivo de los servicios de Salud, Educación, Vivienda, Medios de Comunicación y Trabajo", en todas las localidades y sus áreas de influencia.

Otros aspectos tenidos en cuenta en estos acuerdos se referian, por un lado, al tamaño de la administración; se preparaba un municipio con una planta de personal reducida y eficiente, un ejecutivo con sólo tres secretarias, un consejo deliberante con sólo seis concejales, limitando al máximo la burocratización de la estructura administrativa. Por otro lado, la descentralización administrativa aseguraria la reparación de desigualdades notables entre Verónica, las localidades chicas y las áreas rurales

\section{Indio sano. Un diseño para la salud centrado en la atención primaria}

\subsection{Los problemas de la salud y del sistema en Punta Indio}

La propuesta de salud surge del trabajo conjunto de la comunidad con equipos técnicos especializados y los líderes políticos interesados en el tema de la atención de la salud. En realidad, un enfoque particular del tema de la salud pública estuvo siempre en el centro de las preocupaciones de algunos políticos que están hoy en el gobierno local, y que fueron gestores de la creación del nuevo municipio, dada la formación profesional de los mismos en diversas especialidades médicas.

El lider político de la iniciativa, actual Intendente de Punta Indio (ex Intendente de Magdalena), que por entonces ocupaba una banca en el Senado de la
Legislatura, impulsó la marcha del proyecto de Ley y fue quien, junto a otros lideres locales, promovió abiertamente la movilización y el involucramiento de la comunidad en el proceso. Este punto de partida permite entender los esfuerzos puestos en el programa de salud del municipio. La nueva administración pretendia, en este y en otros ámbitos, "...demostrar que se puede trabajar de otra forma, que el estado municipal puede ser eficiente trabajando para y con la gente, identificando sus problemas y dando respuestas" (Colabianchi, 2000).

En lo relativo a la salud, su amplia actuación política y profesional en la provincia de Buenos Aires le permitía ver con preocupación, por un lado, "...el avance masivo y corporativo de la medicina centrada en la enfermedad"; siendo esta una alternativa cara, basada en el núcleo diagnóstico/ aparatología/tratamiento en instituciones/medicina de gran complejidad era, sin embargo, la que se imponía en el sistema público, cada vez más cercado por la escasez de recursos. Por otro lado, observaba "... el gran descuido, olvido, casi abandono de la medicina preventiva y de las acciones en el nivel primario de atención, en donde se practica una medicina centrada en la preservación de la salud, este nivel de atención necesita menores inversiones y en el largo plazo se resuelven muchos problemas con gran economía de recursos, este tema de los recursos es muy importante si se quiere trabajar con eficiencia en la administración".

Los problemas básicos que corresponde plantear, entiende el Dr. Colabianchi, remiten a cómo se entienden la salud y la enfermedad en la sociedad y a cuál es 
el papel que tienen los involucrados en este campo, hay que preguntarse cómo se forma en las universidades e instituciones especializadas a los profesionales del equipo de salud. Se forman profesionales para instituciones del sistema estatal/público y del sistema privado (hospital, clinica, consultorio, etc.), personal que es valorado en la medida de una progresiva especialización a lo largo de su trayectoria profesional. Desde esta perspectiva, el sujeto/enfermo es tomado por la medicina y recibe las prácticas en una situación de completa pasividad. No hay una formación orientada a la medicina preventiva, que además aparece desvalorizada entre la mayoría de los profesionales que la consideran una "medicina para pobres". En este sentido, "... las corporaciones médicas, los laboratorios, los proveedores de equipos médicos, tienen un gran negocio, porque se gasta muchísimo en salud cuando el eje está puesto en la enfermedad; sin duda estos intereses no van a permitir un cambio revolucionario, desplazar el eje de la enfermedad a la conservación de la salud, a partir de acciones preventivas, planificadas y reforzadas". Desde esta concepción el sujeto sano tiene un rol activo en la preservación de su salud y la de su comunidad.

Pero, también se destacó que el camino de la atención de la salud apoyándose en la medicina preventiva no es fácil de construir; en primera instancia hay que arrasar con décadas de un modelo imperante muy distinto, que genera toda una serie de conductas difíciles de modificar en la gente (por ej. cuando se produce una enfermedad hay una fuerte presión para que en la consulta se proporcione un tratamiento "tradicional", vale decir con estudios, medicamentos, etc., cuando no todos los casos lo requieren). Para contrarrestar la inercia de este sistema caroe ineficiente y modificar la conducta de la población, el principal camino es la educación; ".... existe una necesidad enorme de educar para la salud, de crear conciencia e implicación en la población, porque una parte importante de los éxitos que se alcanzan con el cambio del modelo de atención, dependen de las actitudes y acciones del sujeto que recibe las prácticas, ahora los sujetos son los protagonistas de una salud que hay que conservar".

La atención primaria, además, revaloriza la persona porque cambia la relación médico-paciente, acercándolos notablemente a través de diversos vínculos. La relación médico-paciente mediatizada por prácticas complejas "... ha descuidado enormemente la contención de los pacientes, tomándolos como objetos de la práctica médica en lugar de considerarlos como personas (lo físico y lo psiquico) con problemáticas diversas que se enferman".

Con toda claridad se entendia que el nuevo modelo de atención centrado en la salud debia construirse para la gente y con la gente, que al involucrarse en las acciones lo tomaría como propio y aseguraría su implementación exitosa. Por otro lado, este tipo de iniciativas comprometidas con las demandas históricas de la comunidad coincidian con la idea de "hacer política con la gente", que guiaba las acciones de los líderes locales.

¿Cuáles eran estas demandas históricas en lo relativo a la salud pública a las que hacemos referencia? La percepción de los vecinos de la nueva administración en relación con los servicios de salud que brindaba el sistema público era 
la de desatención generalizada, en la localidad cabecera; la ciudad de Verónica contaba con una Unidad Sanitaria de la red provincial, que tenia infraestructura obsoleta, recursos mínimos de personal y falta crítica de insumos. A la insuficiencia de atención primaria se sumaba la falta de servicio hospitalario, concentrado en la ciudad de Magdalena (viejo hospital de la red provincial hoy transferido al municipio), de alli provenia el médico que atendía 3 veces por semana, unas pocas horas a la población de la zona. Cualquier urgencia o complejidad relativa implicaba el traslado a Magdalena.

Esta situación se transformaba en abandono por inexistencia de servicios públicos para las localidades chicas, Las Pipinas, Punta Indio, Alvarez Jonte y para las zonas rurales (parajes La Viruta, Las Tahonas, Monte Veloz, Punta Piedras y Luján del Rio). Para esta población la falta de conexión fluida con las ciudades más grandes, la propia Verónica y Magdalena agudizaba el problema. En este sentido, la infraestructura vial es una deuda histórica que reciaman los residentes, no hay caminos asfaltados entre las localidades pequeñas y Verónica (salvo Pipinas) y, aún hoy, solo hay un tramo asfaltado entre Verónica y Magdalena.

La oferta del sector privado, al que sólo algunos podian acceder (a través de obras sociales o sistemas de prepago) completaba el panorama de los servicios de salud en el nueva municipio.

Frente a esta situación, ¿que pedía la comunidad? Un sistema público que garantizara la presencia de médicos en forma permanente en el municipio; la demanda de la gente apuntaba a la vieja figura del médico de cabecera arraigado en la comunidad.

Una vez diseñado el sistema de salud para el nuevo municipio, la implantación requeria recursos económicos importantes y profesionales capacitados. Los recursos genuinos para la salud se generan a partir una redistribución de gastos y de un incremento del $10 \%$ en las contribuciones municipales que se cobran a los habitantes del municipio. Tal como lo especifican las normas vigentes, este "aumento de impuestos" debió ser aprobado por la Asamblea de Grandes Contribuyentes y el Consejo Deliberante. Esta medida, impopular como pocas, es aceptada por la población, sin mayores resistencias, en este contexto de verdadero involucramiento en las acciones de la gestión ${ }^{7}$.

\subsection{La atención primaria de la salud en el "Indio Sano". Algunos detalles del funcionamiento del sistema}

El Sistema Municipal de Salud Pública Universal Indio Sano, que se sostiene con recursos propios, está compuesto por distintos subprogramas. Articula un proyecto innovador de origen local surgido de la demanda de la comunidad, el "médico y enfermera de familia", que constituye el subprograma central/básico de todo el sistema, con la oferta clásica de recursos de origen provincial, que se 
organiza en un conjunto amplio de otros subprogramas parcialmente integrados en el del "medico/enfermera de familia".

Los mayores esfuerzos del área de salud están concentrados en el subprograma Médico/Enfermera de Familia que insume entre el 60 y el $70 \%$ de los recursos del presupuesto del área (aprox. 1.300.000 pesos anuales)

Este subprograma núcleo depende de la absoluta responsabilidad de 6 médi$\cos$ y 12 enfermeras de familia, los responsables del área de salud estiman que, con este número de profesionales y con la coordinación de acciones que ha llegado a concretarse, se está en un buen punto de funcionamiento del sistema y de organización de las actividades. Cada equipo básico compuesto por un médico y 2 enfermeras tiene a su cargo la población de una de las varias áreas programáticas en que se divide al territorio del municipio para la prestación de la atención primaria, atendiendo un total de 200 familias o 1.000 personas. En la ciudad de Veróni$\mathrm{ca}$, que concentra una parte significativa de la población total, las áreas programáticas son lo suficientemente flexibles como para respetar en parte las preferencias de la población, que pudo elegir con qué médico atenderse.

La elección de un médico de familia, no implica la dependencia exclusiva de ese profesional, ya que el sistema funciona por interconsulta entre los médicos y enfermeros que están en contacto permanente; en relación con las patologias a tratar pueden derivarse los pacientes entre ellos, ya que además de la clínica general, van desarrollando áreas de especialización según el propio interés. Este subprograma atiende prioritariamente a la población con acciones integrales para la prevención de la salud que vinculan al equipo médico con personal del sistema educativo y con la comunidad, a través de la acción de los Consejos de Salud o a través de la participación directa.

Por otra parte, garantiza la atención de la enfermedad a través de la consulta en las instituciones o en el domicilio y capta a los pacientes que es necesario derivar al nivel de complejidad que demanda la patología. Los pacientes captados por el médico de familia pueden ser derivados a la atención de otros subprogramas con los que se articulan, a la atención de especialistas en el hospital municipal, etc. También son los que intervienen en las decisiones de hospitalización para las diferentes prácticas.

Este subprograma se articula con todos los otros que integran el sistema, a través de uno de sus médicos residentes. Así, además de la atención de la población del área programática correspondiente, que se organiza de diferentes maneras para garantizar la cobertura permanente en las zonas rurales y urbanas, cada médico tiene a su cargo la responsabilidad de otro subprograma; en general, estos subprogramas corresponden a distintas acciones que en materia de salud impulsa el Ministerio de Salud de la Provincia de Buenos Aires, destinando recursos especiales para su implementación

- Subprograma Municipal de Atención Materno-Infantil.

- Subprograma Municipal para la Reducción del Bajo Peso al Nacer.

- Subprograma Municipal de Normatización y Educación Continua para la Salud de la Infancia (Gurises1 y Gurises2). 
- Subprograma de Anticipación y Detección de Enfermedades Ocultas

- Subprograma Normas de Diagnósticoy Tratamiento Municipal de Salud y Enfermedad.

- Subprograma Vademécum Municipal Básico (Vad. A: consulta externa; Vad. B: internación, especialidades y Vad. C: terapia intensiva, neonatal, pediatria, adultos).

- Subprograma Laboratorio Municipal Básico (Lab. A: consultas ambulatorias, urgencia y prevención; Lab. B: internación; Lab. C: especialidades y Lab. D: terapia neonatal, pediatría, adultos). Anexo: Servicio de Diagnóstico por Imágenes Municipales.

- Subprograma Historia Clínica Familiar Municipal. Formación de veinte agentes municipales de atención (Trabajador de Atención Esencial T.A.E.).

- Educación de Postgrado Indio Sano.

- Sistema de salud mental Indio Sano

- Subprograma de Control de la Tuberculosis.

- Subprograma Indio Rural en Escuelas Rurales.

- Hospital Municipal. Anexo: El Sistema de Salud Mental Indio Sano

Los programas de origen provincial, implican recursos a los que el municipio no quiere renunciar pero, los toma y adapta a las particularidades del sistema que implementa, centrado en la atención primaria, con gran énfasis en la educación, atención y preservación de la salud. Por lo tanto, aprovechan intensivamente y mejoran los recursos que permiten acciones de difusión del cuidado de la salud. Aquí aparece, básicamente la población trabajando en el sistema.

\section{Conclusiones}

Como ya hemos señalado, la salud pública estuvo siempre en el centro de las preocupaciones de los políticos que están hoy en el gobierno local, los gestores de la creación del nuevo municipio. El líder político de la iniciativa, actual Intendente de Punta Indio que ha renovado su mandato por segunda vez, promovió desde un principio, la movilización y la participación, en consonancia con una forma de entender la política local, "trabajar para la gente", en un marco en el que la administración atiende sus problemas y "trabaja con la gente", entendiendo que la propia comunidad es la más indicada para identificar sus problemas y establecer prioridades. En este sentido, el trabajo durante las "Jornadas Programáticas para el Nuevo Municipio", colocó a la salud en el centro del interés comunitario.

En una larga entrevista, el Dr. Colabianchi destacó que el enfoque de la medicina centrada en la enfermedad que se imponia en el sistema público, tenía que ser desactivado; es un modelo caro que depende de los profesionales y de instituciones públicas $y / 0$ privadas, en donde sólo hay enfermos que tienen un rol absolutamente pasivo. Desde la medicina preventiva y a través de acciones en el nivel primario de atención, se practica una medicina centrada en la preservación de la salud; desde esta concepción el sujeto sano tiene un rol activo en la preservación de su salud y la de su comunidad a la que se le garantizó por diversos mecanismos una participación abierta y plena.

Pero, también se destacó que el camino de la medicina preventiva no es fácil de construir, en primera instancia hay que 
vencer las inercias de lo que está funcionando (conjunto de prácticas profesionales y sistemas de relaciones con roles fuertemente establecidos). El nuevo enfoque que cambia la relación médico-paciente, acercándolos a través de diversos vínculos, revaloriza notablemente a los sujetos de las prácticas al considerarlos como personas (lo físico y lo psíquico) con problemáticas diversas que se enferman".

Una parte importante de los éxitos de la implementación del programa está atado a las modificaciones que se operen en esta dirección; la educación aparece como "la alternativa" para contrarrestar un estado del sistema y cambiar conductas. El modelo de atención centrado en la salud debia construirse con la gente que al involucrarse en las acciones lo tomaría como propio y aseguraría su implementación exitosa.

En la primera etapa del diseño del modelo de salud, la participación se canalizó a través de la participación directa en asambleas y comisiones de trabajo permanentes, que se consolidan en los Consejos Locales/Federal de Salud y en la Banca Honoraria, institucionalizados por Ordenanza.

El nuevo modelo de salud ambicioso en una cobertura mucho mayor a la existente en el municipio (contemplaba por ej. el reacondicionamiento integral de la infraestructura de la Unidad Sanitaria de Verónica, la única existente y la creación de Unidades Sanitarias en Pipinas y Punta Indio y del Hospital Municipal en Verónica, como centro de mayor complejidad), dependía críticamente de los recursos que lo financiarian. El municipio, a tra- vés de una diferente asignación de partidas y la propia población, que acepta un aumento en las tasas y contribuciones, son los sostenes genuinos del sistema.

Por último, una vez establecidos los subprogramas y ajustado su funcionamiento, la comunidad tiene espacios de participación más definidos, vinculados prioritariamente con la educación para la salud; un cierto control del funcionamiento del sistema (por ej. el rescate y observancia de los enfermos, la alerta sobre situaciones de riesgo y un espacio de trabajo comunitario tal como huertas, talleres y otras actividades que han surgido de los vinculos creados en el marco del proceso de construcción del Indio Sano.

A lo largo de todo este tiempo, la participación que fue ampliamente reclamada por la población cristalizó en grandes logros; pero dado que la motivación es un fenómeno complejo que cuesta mantener sin alteraciones a lo largo del tiempo; la participación ha decaído. Hoy, las autoridades y los equipos de salud reconocen que se ha pasado de una situación de máximo involucramiento y enorme compromiso, a otra de "relativo alejamiento", imputable, por un lado a un buen funcionamiento del sistema y por otro, una cierta dependencia que se ha creado entre la comunidad y el gobierno local que "soluciona todo los problemas", produciendo nuevas inercias que, paradójicamente, restan espacio a la participación.

\section{Bibliografía citada}

Bertini, S. (1995). "Proyectos participativos comunitarios. Dificultades encontradas en su implementación a nivel municipal". Ponencia presentada en la Jornada: 
La problemática urbana en las grandes ciudades. Buenos Aires: C.E.A.

Bustelo Graffigna, E. (1995). "El abrazo. Reflexiones sobre las relaciones entre el estado y los organismos no gubernamentales". En ENOIKOS, Año III, N 9, dic.

Cravacuore, D.; Fernández, M.; llari, S. y A. Villar (1998). Guía de Programas Sociales de la Provincia de Buenos Aires. Versión definitiva actualizada a marzo. UNO.

Estadística Bonaerense (1996). La Plata (Buenos Aires): Dirección Provincial de Estadistica.

Colabianchi, L. y J. Reichenbach (1997). Indio Sano. Una Construcción Comunitaria de la Salud. Verónica (Buenos Aires): Ediciones del Sur.

Fernăndez, M. (1997). "El Programa Matemo infantil y Nutricional (PROMIN). Algunos aspectos de su implementación en el Partido de Berazategui". UNQ. Mimeo.

Garcia Delgado, D. (comp.) (1997). Hacia un nuevo modelo de gestión local. Municipio y Sociedad Civil en Argentina. Buenos Aires: CBC-UBA.

Groisman, E. (1988). "Discutiendo el problema de la participación". En: El Bimestre Político y Económico, № 39, Buenos Aires.

Kliksberg, B. (1992). Cómo será la gerencia en la década del 90. Buenos Aires: Gerencia Social.
Legislatura de la Provincia de Buenos Aires (1994). Ley de Creación del Partido (Municipio) . La Plata. Argentina.

Licciardi, Ana (2000). "El Programa Indio Sano. Una respuesta comunitaria para la Política Sanitaria de un Nuevo Municipio". En Cravacuore, D y G. Badia (compiladores). Experiencias Positivas en Gestión Local. Bernal (Buenos Aires): Universidad Nacional de Quilmes.

Martinez Nogueira, R. (1995). "El Tercer Sector. ¿Por qué y para qué?". En ENOlKOS, Año III, № 9, dic.

Mendicoa, G. (1997). La planificación de las políticas sociales. Buenos Aires: Espacio Editorial Capítulo II, pp. 73-109.

Ministerio de Salud de la Provincia de Buenos Aires (1996). Resolución Ministerial 1946/1996. La Plata. Argentina.

Passalacqua, E. y A. Villar (1994). "La política social de los municipios argentinos: un panorama global". En Revista de Ciencias Sociales, № 1, Universidad Nacional de Quilmes. Quilmes.

Pirez, P. (1991), Municipio, necesidades sociales y política local, Buenos Aires: GEL.

Universidad de Quilmes (2000). Banco de Experiencias Locales, http://www.unq. edu.ar/bel.

Victory, C. (1997). Gobiernos Municipales y Desarrollo Local en Latinoamérica. Naciones Unidas/CEPALILPES. Dirección de Desarrollo y Gestión Local. Documento de distribución restringida (LC/IP/R 201). Chile. 